\title{
Score for the Overall Survival Probability Scores of Fibrosarcoma Patients after Surgery: A Novel Nomogram-Based Risk Assessment System
}

\author{
Yuyuan Chen, ${ }^{1,2}$ Changxing Chi, ${ }^{3}$ Dedian Chen, ${ }^{2}$ Sanjun Chen, ${ }^{4}$ Binbin Yang, ${ }^{5}$ Sijia Huang, \\ and Zengpai Zheng $\mathbb{D}^{1}$ \\ ${ }^{1}$ Department of Anorectal Surgery, The People's Hospital of Pingyang, Wenzhou, Zhejiang, China \\ ${ }^{2}$ The Second Department of Breast Surgery, The Third Affiliated Hospital of Kunming Medical University, Kunming, \\ Yunan, China \\ ${ }^{3}$ Department of Radiation Oncology, The Third Affiliated Hospital of Kunming Medical University, Kunming, Yunan, China \\ ${ }^{4}$ Department of Pain Treatment, The First People's Hospital of Chenzhou, Chenzhou, Hunan, China \\ ${ }^{5}$ Wenzhou Medical University, Wenzhou, Zhejiang, China \\ ${ }^{6}$ Shuitou Town Center Health Center, Wenzhou, Zhejiang, China
}

Correspondence should be addressed to Zengpai Zheng; zhengzengpai@126.com

Received 10 August 2021; Accepted 1 December 2021; Published 22 December 2021

Academic Editor: Shinji Miwa

Copyright $(2021$ Yuyuan Chen et al. This is an open access article distributed under the Creative Commons Attribution License, which permits unrestricted use, distribution, and reproduction in any medium, provided the original work is properly cited.

Background. The primary purpose of this study was to determine the risk factors affecting overall survival (OS) in patients with fibrosarcoma after surgery and to develop a prognostic nomogram in these patients. Methods. Data were collected from the Surveillance, Epidemiology, and End Results database on 439 postoperative patients with fibrosarcoma who underwent surgical resection from 2004 to 2015. Independent risk factors were identified by performing Cox regression analysis on the training set, and based on this, a prognostic nomogram was created. The accuracy of the prognostic model in terms of survival was demonstrated by the area under the curve (AUC) of the receiver operating characteristic curves. In addition, the prediction consistency and clinical value of the nomogram were validated by calibration curves and decision curve analysis. Results. All included patients were divided into a training set $(n=308)$ and a validation set $(n=131)$. Based on univariate and multivariate analyses, we determined that age, race, grade, and historic stage were independent risk factors for overall survival after surgery in patients with fibrosarcoma. The AUC of the receiver operating characteristic curves demonstrated the high predictive accuracy of the prognostic nomogram, while the decision curve analysis revealed the high clinical application of the model. The calibration curves showed good agreement between predicted and observed survival rates. Conclusion. We developed a new nomogram to estimate 1-year, 3-year, and 5-year OS based on the independent risk factors. The model has good discriminatory performance and calibration ability for predicting the prognosis of patients with fibrosarcoma after surgery.

\section{Introduction}

Fibrosarcoma is a group of malignant soft tissue tumors. As solid tumors composed of fibroblasts, FS often involve the deep soft tissues of the extremities, trunk, head, and neck. Surgery is the primary treatment of fibrosarcoma, but the prognosis of its patients varies depending on the type of tumor [1].

Adult fibrosarcoma used to be the most common soft tissue sarcoma. However, with the increased awareness of soft tissue tumors and fibrosarcoma, the diagnosis of adult fibrosarcoma has been tightly defined so that its incidence may be as low as $1 \%$ of adult soft tissue sarcomas $[2,3]$. Unlike the highly malignant adult fibrosarcoma, congenital or infantile fibrosarcoma is intermediate in 2020 WHO classification, with few metastases and a better prognosis [4-6]. Since neurotrophic tropomyosin receptor kinase (NTRK) fusion often occurs in infantile fibrosarcoma, the application of NTRK inhibitors such as larotrectinib is highly efficacious in patients [7-9]. Dermatofibrosarcoma 
protuberans (DFSP) is a low-grade, growing by infiltration but highly locally recurrent fibroblastic malignancy. DFSP most commonly occurs in middle-aged and young adults. Through surgery, radiation therapy, or the targeted drug imatinib mesylate, patients with DFSP have a good prognosis and a high survival rate [10-12]. Surgical resection is the standard treatment for most patients, whether it is a typical or a particular type of fibrosarcoma. Therefore, the evaluation of postoperative prognostic indicators of fibrosarcoma will provide valuable guidance for the clinical treatment of fibrosarcoma.

Nomograms integrate multiple risk indicators and are often used to predict disease survival [13-15]. Fibrosarcoma is primarily treated surgically but has a high recurrence rate after simple resection $[2,16]$. Recently, Xiang et al. constructed a nomogram of 663 cases of fibrosarcoma and determined that age, sex, surgical use, tumor stage, pathologic grade, and tumor size may affect cancer-specific survival [15]. However, studies related to predicting overall survival (OS) after surgery in patients with fibrosarcoma have not been reported. This study aimed to develop a nomogram to identify the clinical and pathological factors associated with improved OS in fibrosarcoma patients after surgery using the Surveillance, Epidemiology, and End Results (SEER) database.

\section{Methods}

2.1. Inclusion and Exclusion Criteria of Patients. The SEER database is updated annually with the latest cancer information. We identified patients with fibrosarcoma from 2004 to 2015 by the SEER * Stat version 8.3.9. The inclusion criteria were as follows: (1) histology ICD-O-3 was limited to $8810 / 3,8812 / 3,8813 / 3,8814 / 3,8823 / 3,8832 / 3,8833 / 3,9321 /$ 3 , and $9330 / 3$; (2) fibrosarcoma as the patient's primary tumor. The exclusion criteria were as follows: (1) the detailed information lacks age, race, grade, primary site, tumor size, and marital status; (2) unknown historic stage and information related to treatment (radiotherapy and chemotherapy). The final dataset we screened had 439 patients with fibrosarcoma. All patients were randomly divided into a training set $(70 \%)$ and a validation set (30\%) for constructing and validating the nomogram.

2.2. Variable Declaration. The age was regrouped as $<60$ years old (young) and $\geq 60$ years old (old). The race was categorized as white, black, and others. The primary site of the tumor was classified as the head and neck, trunk, and extremities. Tumor size was reclassified as $<50$ and $\geq 50 \mathrm{~mm}$. Information on radiotherapy was divided into no radiotherapy, preoperative radiotherapy, and postoperative radiotherapy.

2.3. Statistical Analysis. The primary endpoint of this study, overall survival, was analyzed by Kaplan-Meier survival curves, and a log-rank test for significance was performed [14]. Prognostic factors with a $P$ value $<0.05$ in univariate Cox regression analysis were included in multivariate Cox regression analysis. In multivariate Cox regression analysis, $P$ values $<0.05$ were considered statistically significant, indicating that the variables examined were independent risk factors. In addition, hazard ratios and 95\% confidence intervals were used to adjust prognostic variables.

Based on the above results, we plotted the nomogram against the training set by the rms package. The discrimination of the nomogram was demonstrated by the area under the curve (AUC) of the time-dependent receiver operating characteristic (ROC) curves. In addition, 1-year, 3-year, and 5-year calibration curves and decision curve analysis (DCA) were generated to validate the nomogram's predictive consistency and clinical value. Through the risk assessment system established by $X$-tile software, the patients with fibrosarcoma after surgery were classified into high-risk, intermediate-risk, and low-risk groups, further demonstrating the application value of the predictive model. All statistics were analyzed by $R$ software (version 4.0.3), while $P<0.05$ (two-sided) was considered significant.

\section{Results}

3.1. Baseline Characteristics of the Patients. A total of 439 patients with fibrosarcoma after surgery from the SEER database were included according to our criteria. In addition, 308 patients were included in the training set, and 131 patients were included in the validation set. As given in Table 1, the proportion of male patients was higher than that of females ( $54.5 \%$ vs. $45.5 \% ; P=0.218)$. The median age of the primary diagnosis was $52(25-75 \%, 37-65)$. Most patients were white $(67.9 \%)$, and $65.3 \%$ were $<60$ years old. $7.5 \%$ of the population had primary disease sites in the head and neck, $43.2 \%$ in the trunk, and $49.4 \%$ in the extremities. $35.1 \%$ of screened patients with postoperative fibrosarcoma were in stage I, $31.5 \%$ in stage II, $17.5 \%$ in stage III, and $15.9 \%$ in stage IV. $9.7 \%$ of the patients received chemotherapy, and $30.5 \%$ received radiation therapy, of which $3.6 \%$ were preoperative and $26.9 \%$ were postoperative. The majority of patients were localized (75.6\%), and most tumors were $\geq 50 \mathrm{~mm}$ (55.5\%).

\subsection{Risk Factors in Postoperative Prognosis of Fibrosarcoma.} To identify factors that may predict the occurrence of postoperative fibrosarcoma, univariate and multivariate analyses were performed on the training set. As given in Table 2, the age, race, grade, chemotherapy, tumor size, and historic stage were strongly associated with the OS of the patients with fibrosarcoma after surgery. Consistent with univariate analysis, Kaplan-Meier analysis also showed that clinical factors (age, race, grade, chemotherapy, tumor size, and historic stage) were significantly associated with OS (Figure 1). The multivariate Cox regression analysis confirmed that age, race, grade, and historic stage were independent risk factors (Table 2).

3.3. Development and Validation of Nomogram. We integrated multiple predictors to make a nomogram based on Cox regression analysis to express the interrelationship 
TABle 1: Characteristics of patients with postoperative fibrosarcoma.

\begin{tabular}{|c|c|c|c|c|c|c|}
\hline \multirow{2}{*}{ Variables } & \multicolumn{2}{|c|}{ Total set } & \multicolumn{2}{|c|}{ Training set } & \multicolumn{2}{|c|}{$\begin{array}{c}\text { Validation } \\
\text { set }\end{array}$} \\
\hline & $\begin{array}{c}N=439 \\
n\end{array}$ & $\%$ & $\begin{array}{c}N=308 \\
n\end{array}$ & $\%$ & $\begin{array}{c}N=131 \\
N\end{array}$ & $\%$ \\
\hline \multicolumn{7}{|l|}{ Age } \\
\hline$<60$ & 292 & 66.5 & 201 & 65.3 & 91 & 69.5 \\
\hline$\geq 60$ & 147 & 33.5 & 107 & 34.7 & 40 & 30.5 \\
\hline \multicolumn{7}{|l|}{ Race } \\
\hline Black & 92 & 21 & 65 & 21.1 & 27 & 20.6 \\
\hline Others & 40 & 9.1 & 34 & 11 & 6 & 4.6 \\
\hline White & 307 & 69.9 & 209 & 67.9 & 98 & 74.8 \\
\hline \multicolumn{7}{|l|}{ Sex } \\
\hline Female & 201 & 45.8 & 140 & 45.5 & 61 & 46.6 \\
\hline Male & 238 & 54.2 & 168 & 54.5 & 70 & 53.4 \\
\hline \multicolumn{7}{|l|}{ Marital status } \\
\hline Married & 220 & 50.1 & 151 & 49 & 69 & 52.7 \\
\hline Unmarried & 219 & 49.9 & 157 & 51 & 62 & 47.3 \\
\hline \multicolumn{7}{|l|}{ Primary site } \\
\hline Head and neck & 39 & 8.9 & 23 & 7.5 & 16 & 12.2 \\
\hline Trunk & 191 & 43.5 & 133 & 43.2 & 58 & 44.3 \\
\hline Extremity & 209 & 47.6 & 152 & 49.4 & 57 & 43.5 \\
\hline \multicolumn{7}{|l|}{ Grade } \\
\hline I & 142 & 32.3 & 108 & 35.1 & 34 & 26 \\
\hline II & 133 & 30.3 & 97 & 31.5 & 36 & 27.5 \\
\hline III & 86 & 19.6 & 54 & 17.5 & 32 & 24.4 \\
\hline IV & 78 & 17.8 & 49 & 15.9 & 29 & 22.1 \\
\hline \multicolumn{7}{|l|}{ Radiotherapy } \\
\hline No & 302 & 68.8 & 214 & 69.5 & 88 & 67.2 \\
\hline RPS & 14 & 3.2 & 11 & 3.6 & 3 & 2.3 \\
\hline RAS & 123 & 28 & 83 & 26.9 & 40 & 30.5 \\
\hline \multicolumn{7}{|l|}{ Chemotherapy } \\
\hline No & 389 & 88.6 & 278 & 90.3 & 111 & 84.7 \\
\hline Yes & 50 & 11.4 & 30 & 9.7 & 20 & 15.3 \\
\hline \multicolumn{7}{|l|}{ Tumor size } \\
\hline$<50$ & 200 & 45.6 & 137 & 44.5 & 63 & 48.1 \\
\hline$\geq 50$ & 239 & 54.4 & 171 & 55.5 & 68 & 51.9 \\
\hline \multicolumn{7}{|l|}{ Historic stage } \\
\hline Localized & 334 & 76.1 & 233 & 75.6 & 101 & 77.1 \\
\hline Regional & 89 & 20.3 & 65 & 21.1 & 24 & 18.3 \\
\hline Distant & 16 & 3.6 & 10 & 3.2 & 6 & 4.6 \\
\hline
\end{tabular}

RPS, radiation prior to surgery; RAS, radiation after surgery.

between the variables (Figure 2). The tumor grade of fibrosarcoma has the most significant impact on patients, and as tumor grade increases, patients have a worse prognosis. Populations of other races have a better prognosis than those of black or white races. Patients who are $\geq 60$ years old or whose tumors have distant metastases have a worse prognosis.

Next, we examine the prognostic models for the training and validation sets, respectively, by plotting ROC curves (Figure 3). The results have demonstrated that the AUC of the training set is $0.827,0.812$, and 0.814 for 12 months, 36 months, and 60 months, respectively (Figure 3(a)), while the validation set is $0.834,0.817$, and 0.766 , respectively (Figure 3(b)). The DCA can be used to evaluate the net benefit of nomogram-aided decision-making under different threshold probabilities to assess the benefit degree of patients and the clinical application value of the mode [17]. As shown in Figure 4, this nomogram model has good clinical application in predicting the prognosis of fibrosarcoma after surgery. In addition, the calibration curves for both the training and validation sets have identified a strong agreement between the nomogram prediction and realistic observation for 1-year, 3-year, and 5-year of OS (Figure 5).

3.4. Risk Assessment System. The total score for each patient was calculated, and all patients were divided into three groups using X-tile software, including high-risk (>231), intermediate-risk (205-231), and low-risk $(<205)$ groups. Kaplan-Meier survival curves for each risk subgroup were plotted, and the results in both the training and validation sets showed differences in OS for patients with different risk levels $(P<0.0001)$ (Figure 6). While patients with high-risk scores had the lowest survival rate, those with low-risk scores had the highest survival rate, indicating that this risk grouping system has a strong predictive value for the prognosis of patients with fibrosarcoma after surgery.

\section{Discussion}

SEER is a typical medical database that provides systematic evidence support and valuable first-hand information for clinicians' practice and medical research $[13,15,18-20]$. Nomogram is conducive to the promotion of personalized medicine and has been proposed to improve disease prediction [21-23]. Wang et al. developed a prognostic nomogram model for small-cell lung cancer patients. They verified that the performance of nomogram was better than that of early models, including those using AJCC staging [24]. As a pictorial representation of a complex mathematical formula, nomograms respond to our exploration of comprehensive biological and clinical models [17, 22, 23].

Fibrosarcoma is a malignant tumor derived from mesenchymal cells, which is usually invasive and has a high postoperative recurrence rate [16]. Adult fibrosarcoma occurs most frequently in the trunk and limbs, followed by the head and neck, consistent with our results [25]. Tumor size of malignant fibrous neoplasms (MFN) of long bones $>10 \mathrm{~cm}$ is a poor prognostic factor for OS and cancer-specific survival [26]. A report on primary intracranial fibrosarcoma suggested that large tumor volume $(\geq 5 \mathrm{~cm})$ and high Ki-67 index $(\geq 30 \%)$ were independent risk factors for OS $[27,28]$. Like most types of fibrosarcoma, renal fibrosarcoma is highly malignant and has a poor prognosis. Radical nephrectomy is the primary clinical treatment strategy $[29,30]$. Mitotic activity and Ki-67 positive cells were identified as important factors in diagnosing ovarian fibrosarcoma [31, 32]. Less than $5 \%$ of fibrosarcoma originated in the genitourinary tract, and fibrosarcoma of the penis is even rarer. To our knowledge, there are few reports on the prognosis of patients with fibrosarcoma. Xiang et al. demonstrated that age, gender, operation, tumor stage, pathological grade, and tumor size might be independent risk factors affecting the survival of FS patients, among which age is the main factor 
TABLE 2: Univariate and multivariate Cox regression analyses of patients with postoperative fibrosarcoma.

\begin{tabular}{|c|c|c|c|c|}
\hline \multirow{2}{*}{ Characteristics } & \multicolumn{2}{|c|}{ Univariate analysis } & \multicolumn{2}{|c|}{ Multivariate analysis } \\
\hline & HR $(95 \% \mathrm{CI})$ & $P$ value & HR $(95 \% \mathrm{CI})$ & $P$ value \\
\hline \multicolumn{5}{|l|}{ Age } \\
\hline$<60$ & Reference & & Reference & \\
\hline$\geq 60$ & $3.531(2.236-5.575)$ & $\leq 0.001$ & $2.776(1.688-4.566)$ & $\leq 0.001$ \\
\hline \multicolumn{5}{|l|}{ Race } \\
\hline Black & Reference & & Reference & \\
\hline Others & $0.789(0.238-2.622)$ & 0.699 & $0.209(0.051-0.851)$ & 0.029 \\
\hline White & $2.246(1.079-4.678)$ & 0.031 & $1.161(0.524-2.571)$ & 0.712 \\
\hline \multicolumn{5}{|l|}{ Sex } \\
\hline Female & Reference & & & \\
\hline Male & $1.327(0.846-2.081)$ & 0.218 & & \\
\hline \multicolumn{5}{|l|}{ Marital status } \\
\hline Married & Reference & & & \\
\hline Unmarried & $1.295(0.828-2.026)$ & 0.257 & & \\
\hline \multicolumn{5}{|l|}{ Primary site } \\
\hline Head and neck & Reference & & & \\
\hline Trunk & $0.723(0.314-1.667)$ & 0.447 & & \\
\hline Extremity & $1.083(0.489-2.400)$ & 0.844 & & \\
\hline \multicolumn{5}{|l|}{ Grade } \\
\hline I & Reference & & Reference & \\
\hline II & $7.157(2.490-20.574)$ & $\leq 0.001$ & $8.068(2.756-23.621)$ & $\leq 0.001$ \\
\hline III & $14.008(4.859-40.386)$ & $\leq 0.001$ & $10.298(3.520-30.130)$ & $\leq 0.001$ \\
\hline IV & $18.089(6.311-51.847)$ & $\leq 0.001$ & $14.840(5.060-43.521)$ & $\leq 0.001$ \\
\hline \multicolumn{5}{|l|}{ Radiotherapy } \\
\hline No & Reference & & & \\
\hline Radiation prior to surgery & $1.438(0.518-3.992)$ & 0.485 & & \\
\hline Radiation after surgery & $1.295(0.808-2.077)$ & 0.283 & & \\
\hline \multicolumn{5}{|l|}{ Chemotherapy } \\
\hline No & Reference & & Reference & \\
\hline Yes & $3.429(2.041-5.760)$ & $\leq 0.001$ & $0.879(0.440-1.757)$ & 0.715 \\
\hline \multicolumn{5}{|l|}{ Tumor size } \\
\hline$<50$ & Reference & & Reference & \\
\hline$\geq 50$ & $3.050(1.802-5.160)$ & $\leq 0.001$ & $1.597(0.904-2.821)$ & 0.107 \\
\hline \multicolumn{5}{|l|}{ Historic stage } \\
\hline Localized & Reference & & Reference & \\
\hline Regional & $1.731(1.051-2.851)$ & 0.031 & $1.197(0.712-2.010)$ & 0.498 \\
\hline Distant & $9.271(4.513-19.047)$ & $\leq 0.001$ & $10.556(3.857-28.889)$ & $\leq 0.001$ \\
\hline
\end{tabular}

affecting the prognosis [15]. Surgery is the standard treatment for fibrosarcoma at various sites, but the prognosis is abysmal [33]. A reported case of cavernous fibrosarcoma died ten months after the operation, although he received chemotherapy [34]. Patel et al. analyzed 51 sinonasal fibrosarcoma patients from the SEER database. They found that the disease-specific survival rate of patients treated with surgery was better than patients treated with primary radiotherapy alone $[35,36]$. However, there has been no evaluation of postoperative prognosis in patients with fibrosarcoma.

This study aims to develop a clinically helpful nomogram that could predict the prognosis of patients with fibrosarcoma after surgery. We finally included 439 patients with postsurgical fibrosarcoma from the SEER database. A randomly divided training set was used to establish the prediction model, while the validation set was used to test the model's accuracy. Through univariate and multivariate analyses, we determined that age, race, tumor grade, and historic stage were closely associated with prognosis ( Table 2). We did not find that chemotherapy was an independent prognostic factor for OS, and the results were consistent with a recent study [26]. Based on this, a nomogram was created to facilitate clinical work (Figure 2). Validation is the process of testing models on different groups to obtain unbiased estimates of model performance and judge their applicability to these groups [17]. Knowledge of the identification and calibration of nomograms in this patient group will enable clinicians and patients to comprehensively evaluate the reliability and accuracy of nomograms [17, 37]. Validated by calibration curves, ROC curves, and DCA, our prognostic model showed good internal and external performance in predicting the prognosis of patients with fibrosarcoma after surgery (Figures 3-5).

This study may have limitations due to its retrospective nature. Although the nomogram has good performance, it ignores patients' and doctors' satisfaction and quality of life and lacks clinical utility $[17,38]$. Therefore, all nomograms 

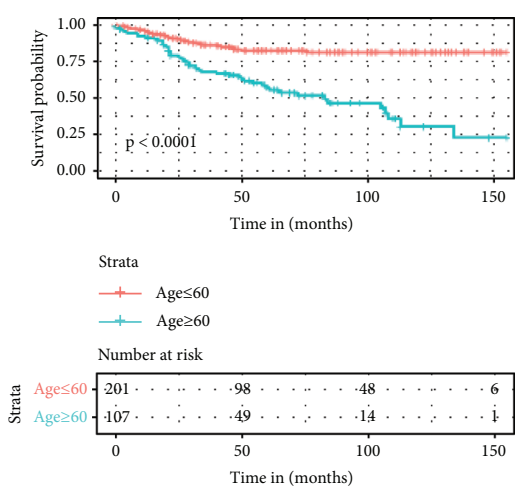

Number of censoring

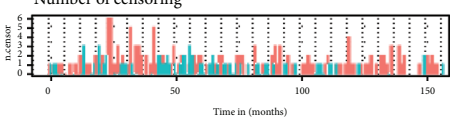

(a)

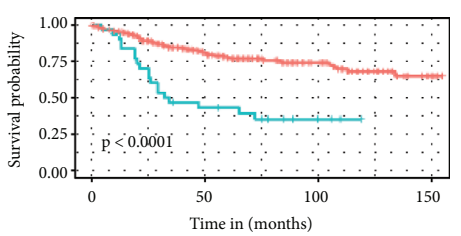

Strata

+ Chemotherapy=No

+ Chemotherapy=Yes

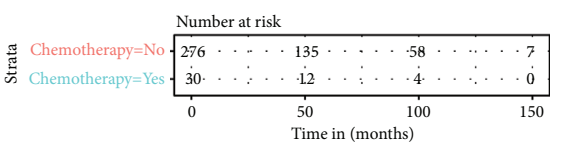

Number of censoring

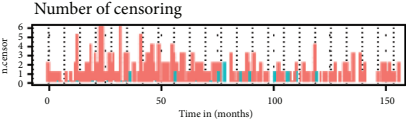

(d)

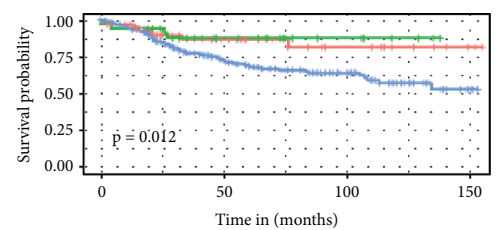

Strata

+ Race=Black

+ Race=Other

+ Race=White

Number at risk

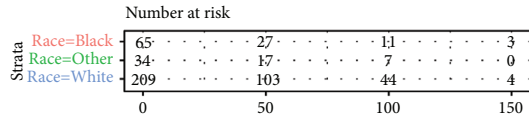

Time in (months)

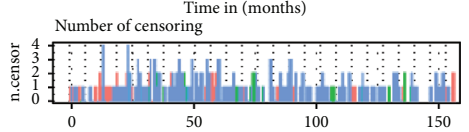

Time in (months)

(b)

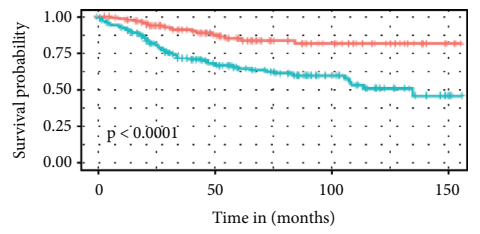

Strata

+ Tumor size $\leq 50$

+ Tumor size $\geq 50$

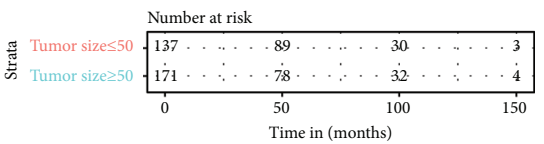

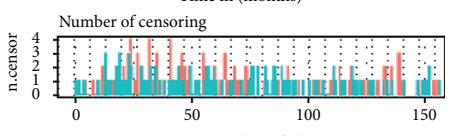

Time in (months)

(e)

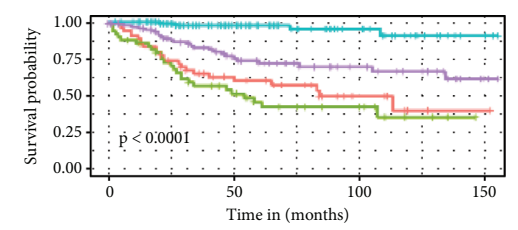

Grade

1 Grade=III — G Grade $=$ I

$\multimap$ Grade=IV —— Grade=II

Number at risk
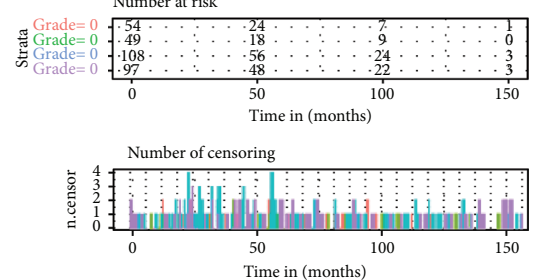

(c)

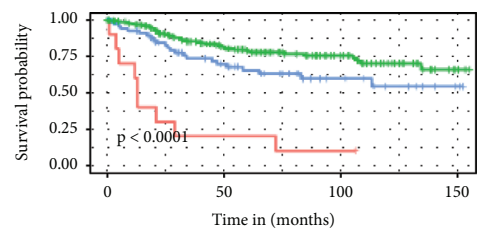

Strata

+ Stage $=$ Distant

+ Stage $=$ Localized

+ Stage=Regional

Number at risk

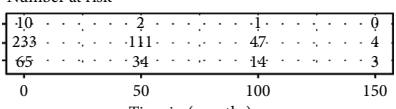

Time in (months)

Number of censoring

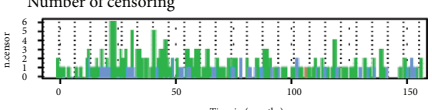

(f)

Figure 1: Kaplan-Meier survival curves of fibrosarcoma patients after surgery stratified by (a) age, (b) race, (c) grade, (d) chemotherapy, (e) tumor size, and (f) historic stage.

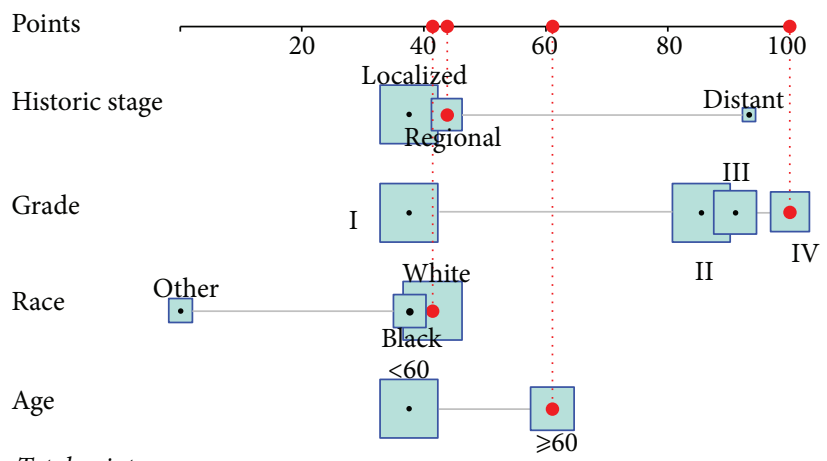

Total points

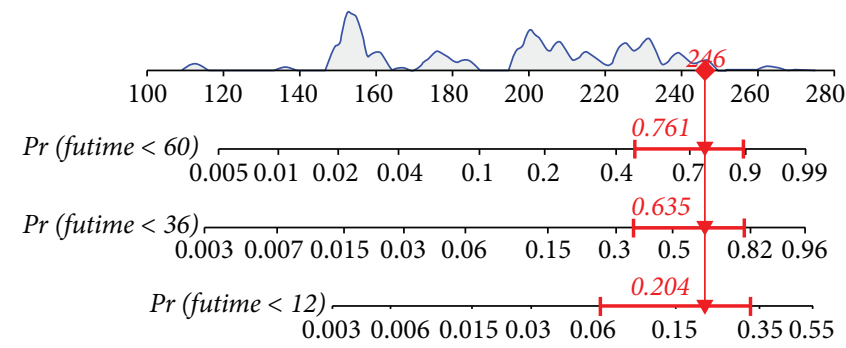

Figure 2: Nomogram for predicting 1-year, 3-year, and 5-year overall survival. The total points were calculated by adding the points of each prognostic factor and correspond to the possibilities of 1-year, 3-year, and 5-year overall survival of fibrosarcoma patients after surgery. 


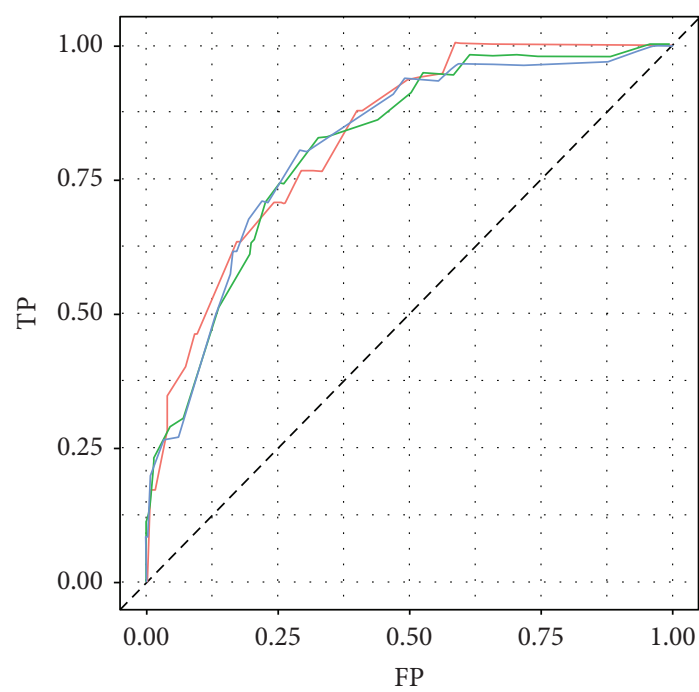

AUC

- 12 Months AUC: 0.827

- 36 Months AUC: 0.812

60 Months AUC: 0.814

(a)

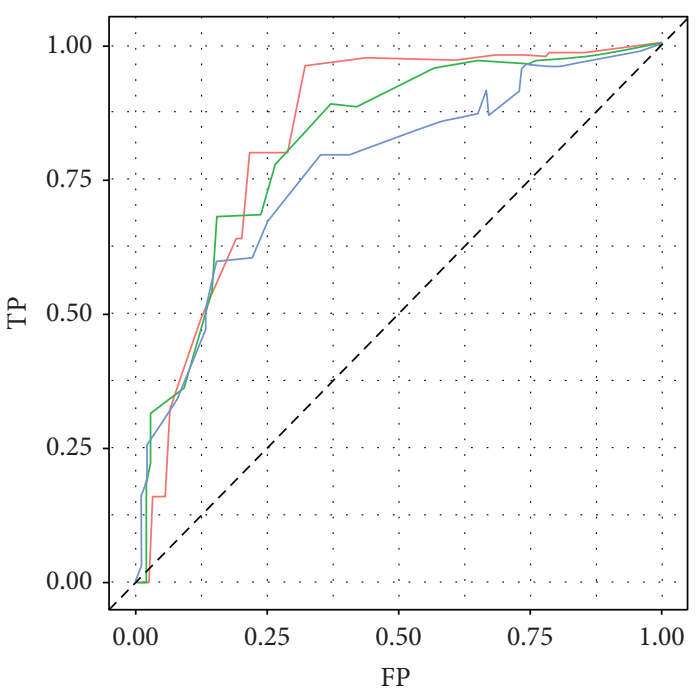

AUC

— 12 Months AUC: 0.834

— 36 Months AUC: 0.817

— 60 Months AUC: 0.766

(b)

FiguRE 3: Receiver operating characteristic curves for predicting 1-year, 3-year, and 5-year overall survival in the training set (a). Receiver operating characteristic curves for predicting 1-year, 3-year, and 5-year overall survival in the validation set (b).
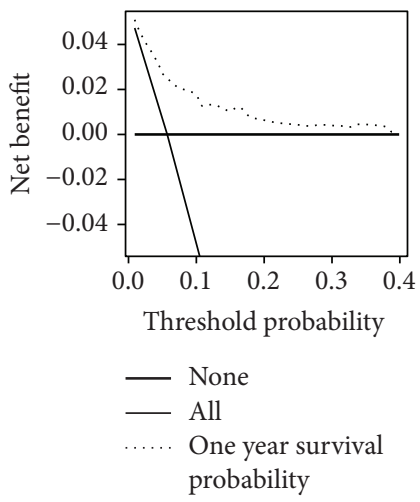

(a)
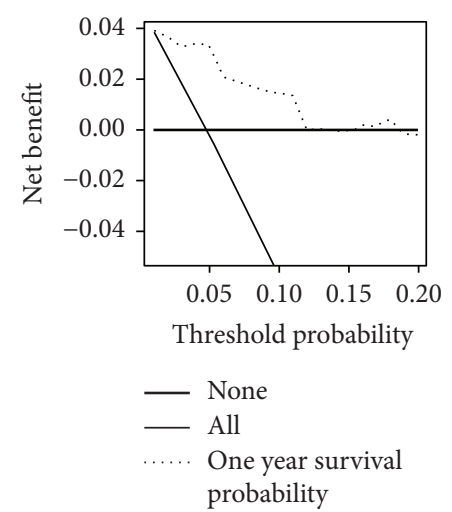

(d)
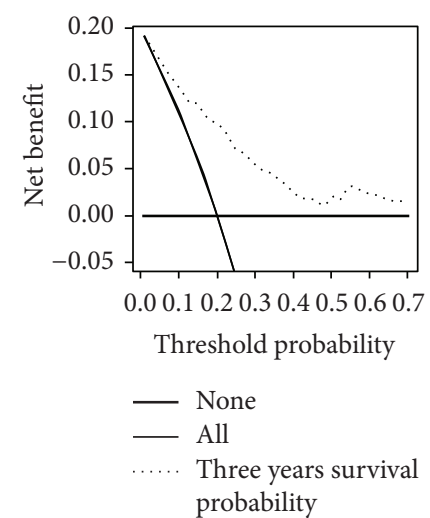

(b)
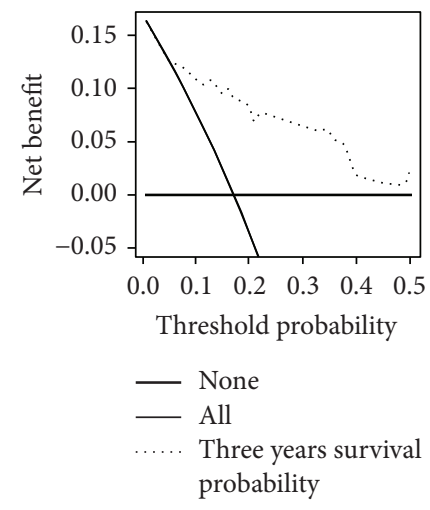

(e)
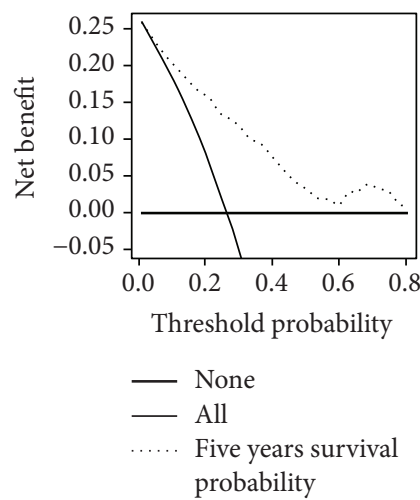

(c)
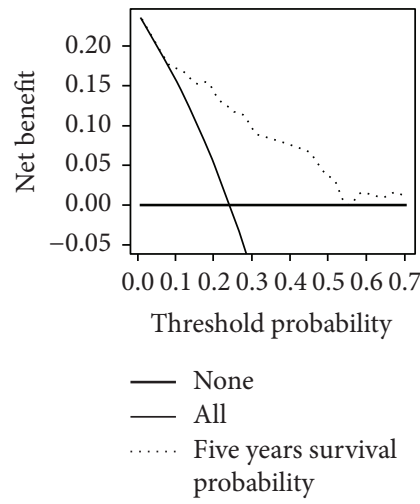

(f)

FIGURE 4: Decision curve analysis of the nomogram for predicting the 1-year (a), 3-year, (b) and 5-year (c) overall survival in the training set and the 1-year (d), 3-year, (e) and 5-year (f) overall survival in the validation set. 


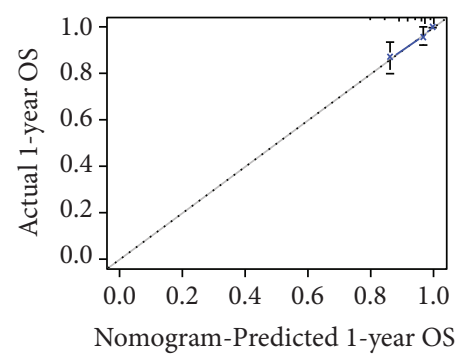

(a)

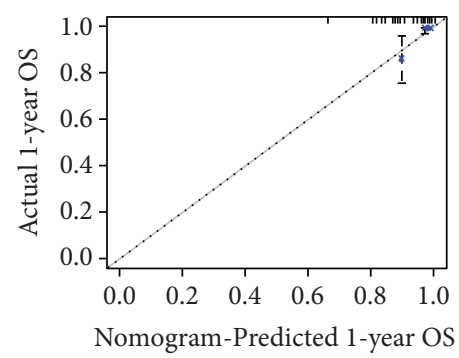

(d)

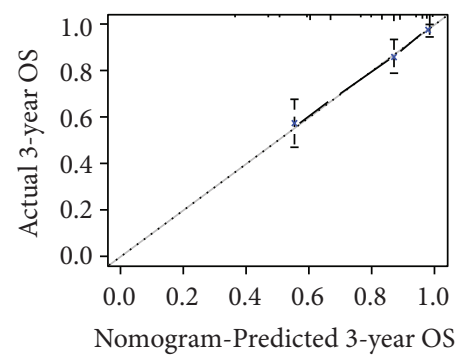

(b)

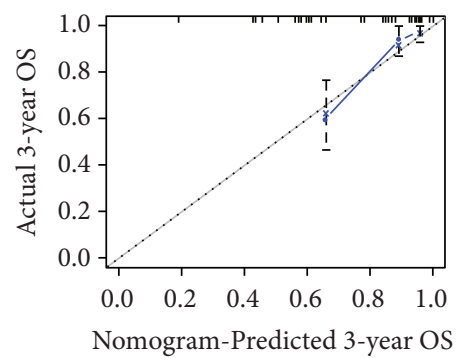

(e)

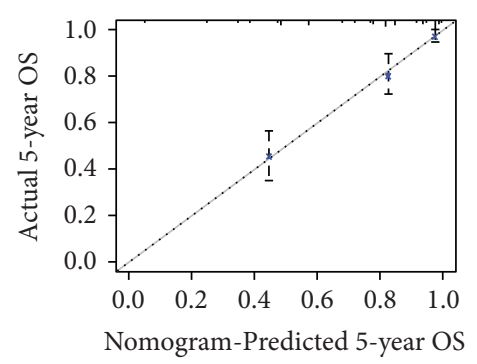

(c)

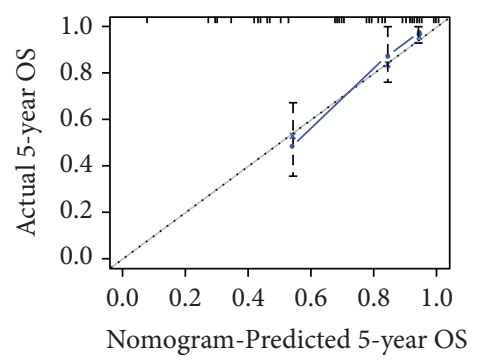

(f)

Figure 5: Calibration curves for 1-year (a), 3-year (b), and 5-year (c) prediction in the training set. Calibration curves for the 1-year (d), 3year, (e), and 5-year (f) prediction in the validation set.

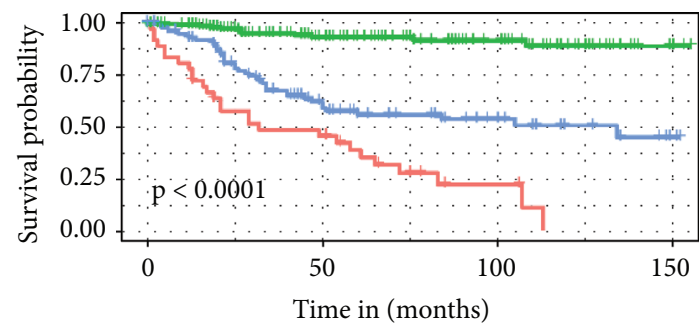

Strata

+ Risk=High

+ Risk=Low

+ Risk=Middle

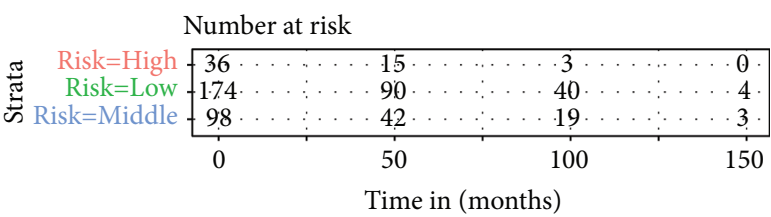

Number of censoring

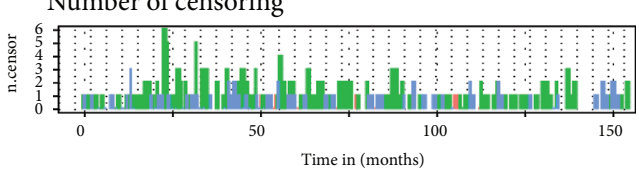

(a)

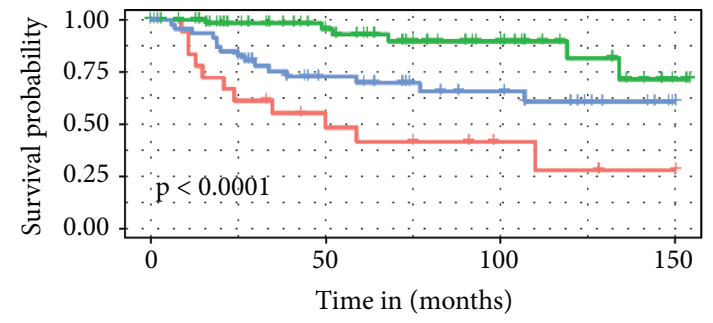

Strata

+ Risk=High

+ Risk=Low

+ Risk=Middle

Number at risk

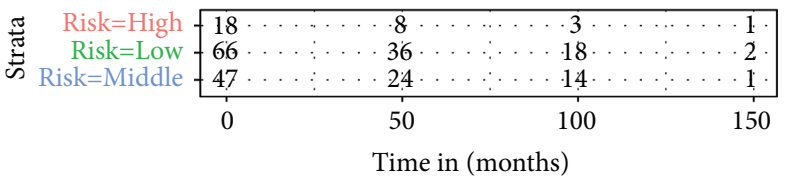

Number of censoring

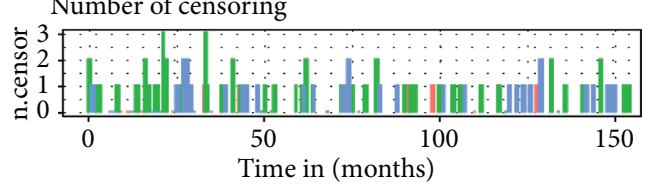

(b)

FIgURE 6: Kaplan-Meier survival analysis for both the training set (a) and the validation set (b). 
require more validation using large independent groups. Nomograms must be rigorously reviewed before they can be used in clinical decision-making. When we understand the performance and limitations of the predictive model, we can provide a better prognosis for patients.

\section{Conclusion}

In this study, we used the SEER database to analyze the prognosis data of patients with fibrosarcoma after surgery for the first time. Nomograms for estimating OS at 1, 3, and 5 years were established based on a large study cohort. The current model has good prediction ability for patient diagnosis, risk assessment, and clinical decision-making to help clinicians provide highly customized patient management in the future.

\section{Abbreviations}

NTRK: Neurotrophic tropomyosin receptor kinase

DFSP: Dermatofibrosarcoma protuberans

OS: Overall survival

SEER: Surveillance, Epidemiology, and End Results

AUC: Area under the curve

ROC: Receiver operating characteristic

DCA: Decision curve analysis.

\section{Data Availability}

The dataset from the SEER database that was generated and/ or analyzed during the current study is available in the SEER dataset repository (https://seer.cancer.gov/).

\section{Ethical Approval}

The data were collected from the SEER database, and due to its retrospective nature, the study was exempted by the SEER database administrators.

\section{Consent}

Not applicable.

\section{Conflicts of Interest}

The authors declare that they have no conflicts of interest.

\section{Authors' Contributions}

YY C, CX C, and ZP Z conceived and designed the study. SJ $\mathrm{C}$ and $\mathrm{BB}$ Y performed the literature search. YY C and SJ H generated the figures and tables. YY Cand CX C analyzed the data and wrote the manuscript. ZP Z critically reviewed the manuscript. YY C and ZP Z supervised the research. Yuyuan Chen, Changxing Chi, and Dedian Chen contributed equally to this work. All authors have read and approved the manuscript.

\section{References}

[1] S. L. Wong, "Diagnosis and management of desmoid tumors and fibrosarcoma," Journal of Surgical Oncology, vol. 97, no. 6, pp. 554-558, 2008.

[2] A. L. Folpe, "Fibrosarcoma: A review and update," Histopathology, vol. 64, no. 1, pp. 12-25, 2014.

[3] H. Wang, P. Nie, C. Dong et al., "CT and MRI findings of soft tissue adult fibrosarcoma in extremities," BioMed Research International, vol. 2018, Article ID 6075705, 7 pages, 2018.

[4] T. Rashid, D. H. Noyd, N. Iranzad, J. T. Davis, and M. D. Deel, "Advances in the diagnosis and management of neonatal sarcomas," Clinics in Perinatology, vol. 48, no. 1, pp. 117-145, 2021.

[5] A. Chandrasekaran, "Neonatal solid tumors," Pediatrics \& Neonatology, vol. 59, no. 1, pp. 65-70, 2018.

[6] T. M. Edwards, M. S. Duran, and T. M. Meeker, "Congenital infantile fibrosarcoma in the premature infant," Advances in Neonatal Care, vol. 17, no. 6, pp. 440-450, 2017.

[7] J. P. Solomon, R. Benayed, J. F. Hechtman, and M. Ladanyi, "Identifying patients with NTRK fusion cancer," Annals of Oncology, vol. 30, no. Suppl_8, pp. viii16-viii22, 2019.

[8] C. M. Albert, J. L. Davis, N. Federman, M. Casanova, and T. W. Laetsch, "TRK fusion cancers in children: A clinical review and recommendations for screening," Journal of Clinical Oncology, vol. 37, no. 6, pp. 513-524, 2019.

[9] T. W. Laetsch, S. G. DuBois, L. Mascarenhas et al., "Larotrectinib for paediatric solid tumours harbouring NTRK gene fusions: phase 1 results from a multicentre, open-label, phase 1/2 study," The Lancet Oncology, vol. 19, no. 5, pp. 705-714, 2018.

[10] X. Hao, S. D. Billings, F. Wu et al., "Dermatofibrosarcoma protuberans: update on the diagnosis and treatment," Journal of Clinical Medicine, vol. 9, no. 6, 2020.

[11] K. Thway, J. Noujaim, R. L. Jones, and C. Fisher, "Dermatofibrosarcoma protuberans: pathology, genetics, and potential therapeutic strategies," Annals of Diagnostic Pathology, vol. 25, pp. 64-71, 2016.

[12] D. DuBay, V. Cimmino, L. Lowe, T. M. Johnson, and V. K. Sondak, "Low recurrence rate after surgery for dermatofibrosarcoma protuberans," Cancer, vol. 100, no. 5, pp. 1008-1016, 2004.

[13] J. Kong, J. Zheng, J. Cai et al., “A nomogram for individualized estimation of survival among adult patients with adrenocortical carcinoma after surgery: a retrospective analysis and multicenter validation study," Cancer Communications, vol. 39, no. 1, p. 80, 2019.

[14] Z. Huang, C. Hu, K. Liu et al., "Risk factors, prognostic factors, and nomograms for bone metastasis in patients with newly diagnosed infiltrating duct carcinoma of the breast: a population-based study," BMC Cancer, vol. 20, no. 1, Article ID 1145, 2020.

[15] G. H. Xiang, J. J. Zhu, C. R. Ke et al., "Nomograms predict overall survival and cancer-specific survival in patients with fibrosarcoma: A SEER-based study," Journal of Oncology, vol. 2020, Article ID 8284931, 9 pages, 2020.

[16] H. Ma, J. Fang, T. Wang et al., "Efficacy and safety of apatinib in the treatment of postoperative recurrence of fibrosarcoma," OncoTargets and Therapy, vol. 13, pp. 1717-1721, 2020.

[17] V. P. Balachandran, M. Gonen, J. J. Smith, and R. P. DeMatteo, "Nomograms in oncology: more than meets the eye," The Lancet Oncology, vol. 16, no. 4, pp. e173-e180, 2015. 
[18] W. Liang, J. He, Y. Shen et al., "Impact of examined lymph node count on precise staging and long-term survival of resected non-small-cell lung cancer: A population study of the US SEER database and a Chinese multi-institutional registry," Journal of Clinical Oncology, vol. 35, no. 11, pp. 1162-1170, 2017.

[19] S. Johnson, M. Renz, L. Wheeler et al., "Vulvar sarcoma outcomes by histologic subtype: a Surveillance, Epidemiology, and End Results (SEER) database review," International Journal of Gynecologic Cancer, vol. 30, no. 8, pp. 1118-1123, 2020.

[20] E. Ward, A. Jemal, V. Cokkinides et al., "Cancer disparities by race/ethnicity and socioeconomic status," CA: A Cancer Journal for Clinicians, vol. 54, no. 2, pp. 78-93, 2004.

[21] D. A. Grimes, "The nomogram epidemic: resurgence of a medical relic," Annals of Internal Medicine, vol. 149, no. 4, pp. 273-275, 2008.

[22] D. Dong, M.-J. Fang, L. Tang et al., "Deep learning radiomic nomogram can predict the number of lymph node metastasis in locally advanced gastric cancer: an international multicenter study," Annals of Oncology, vol. 31, no. 7, pp. 912-920, 2020.

[23] M. W. Kattan, C. Yu, A. J. Stephenson, O. Sartor, and B. Tombal, "Clinicians versus nomogram: predicting future technetium- $99 \mathrm{~m}$ bone scan positivity in patients with rising prostate-specific antigen after radical prostatectomy for prostate cancer," Urology, vol. 81, no. 5, pp. 956-961, 2013.

[24] S. Wang, L. Yang, B. Ci et al., "Development and validation of a nomogram prognostic model for SCLC patients," Journal of Thoracic Oncology, vol. 13, no. 9, pp. 1338-1348, 2018.

[25] A. Bahrami and A. L. Folpe, "Adult-type fibrosarcoma: A reevaluation of 163 putative cases diagnosed at a single institution over a 48-year period," The American Journal of Surgical Pathology, vol. 34, no. 10, pp. 1504-1513, 2010.

[26] Y. Huang, J. Hong, J. Meng et al., "Malignant fibrous neoplasms of long bones: analysis of the surveillance, epidemiology, and end results database from 1973 to 2015," BMC Musculoskeletal Disorders, vol. 21, no. 1, p. 48, 2020.

[27] N. Cai and L. B. Kahn, "A report of primary brain fibrosarcoma with literature review," Journal of Neuro-Oncology, vol. 68, no. 2, pp. 161-167, 2004.

[28] X.-J. Ma, D. Li, H. Li et al., "The clinicoradiological features and surgical outcomes of primary intracranial fibrosarcoma: a single-institute experience with a systematic review," Neurosurgical Review, vol. 44, no. 1, pp. 543-553, 2021.

[29] H. Jiang, L. Liu, and G. Li, "Primary synchronous ipsilateral renal fibrosarcoma and renal pelvic carcinoma: A case report and literature review," OncoTargets and Therapy, vol. 14, pp. 4119-4125, 2021

[30] A. Torabi, J. Corral, Z. Gatalica, J. Swensen, S. Moraveji, and J. A. Bridge, "Primary renal sclerosing epithelioid fibrosarcoma: a case report and review of the literature," Pathology, vol. 49, no. 4, pp. 447-450, 2017.

[31] L. Huang, L.-M. Liao, H.-Y. Wang, and M. Zheng, "Clinicopathologic characteristics and prognostic factors of ovarian fibrosarcoma: the results of a multi-center retrospective study," BMC Cancer, vol. 10, no. 1, p. 585, 2010.

[32] T.-T. Sun, N.-H. Cheng, D.-Y. Cao, and P. Peng, "Ovarian fibrosarcoma: A single-institution experience and a review of the literature," Journal of Ovarian Research, vol. 13, no. 1, p. $142,2020$.

[33] P. Kang, P.-L. Li, J.-Y. Peng, M. Ni, G.-J. Jia, and W. Jia, "Clinical characteristics and surgical features of intracranial fibrosarcoma," Journal of Craniofacial Surgery, vol. 31, no. 3, pp. 825-828, 2020.

[34] Z. Liu and W. Zou, "Fibrosarcoma of the corpus cavernosum: case report and literature review," BMC Surgery, vol. 21, no. 1, p. 20, 2021.

[35] T. D. Patel, E. T. Carniol, A. Vázquez, S. Baredes, J. K. Liu, and J. A. Eloy, "Sinonasal fibrosarcoma: analysis of the surveillance, epidemiology, and end results database," International Forum of Allergy \& Rhinology, vol. 6, no. 2, pp. 201-205, 2016.

[36] J. Zeng, H. Liu, L. Liu et al., "Fibrosarcoma arising in the paranasal sinus: a clinicopathological and radiological analysis," Dentomaxillofacial Radiology, vol. 47, no. 6, Article ID 20170459, 2018.

[37] A. Iasonos, D. Schrag, G. V. Raj, and K. S. Panageas, "How to build and interpret a nomogram for cancer prognosis," Journal of Clinical Oncology, vol. 26, no. 8, pp. 1364-1370, 2008.

[38] M. C. Specht, M. W. Kattan, M. Gonen, J. Fey, and K. J. Van Zee, "Predicting nonsentinel node status after positive sentinel lymph biopsy for breast cancer: clinicians versus nomogram," Annals of Surgical Oncology, vol. 12, no. 8, pp. 654-659, 2005. 